stomatologie 2013 [Suppl 1] · 110:18-18 DOI 10.1007/s00715-013-0264-7

๑) Springer-Verlag Wien 2013

\title{
Grußworte der ÖGZMK Salzburg
}

Liebe Kolleginnen und Kollegen!

Regelmäßige Fort- und Weiterbildung auf wissenschaftlich fundierter Basis ist für unseren Berufsstand unverzichtbar. Die ÖGZMK steht seit 150 Jahren für diese hohe Qualität der beruflichen Weiterentwicklung, die nur durch das Ineinandergreifen von Wissenschaft und Praxis gewährleistet werden kann. Die ÖGZMK ist die größte und traditionsreichste Fortbildungseinrichtung der Zahnärzte in Österreich. Und das aus gutem Grund.

Unter dem starken Dach der ÖGZMK entwickeln die einzelnen Ländergesellschaften und die Fachgesellschaften ihre vielfältigen Aktivitäten und decken damit die gesamte Breite der modernen Zahnheilkunde ab. In Salzburg bieten wir Vorträge namhafter Referenten aus dem Inund Ausland sowie Qualitätszirkel in den Bezirken, die gut besucht sind.

Welch hohen Stellenwert die Fortbildung bei den Kolleg(inn)en hat, bewies der Jubiläumskongress, den die ÖGZMK Salzburg im vergangenen September in der Mozartstadt ausrichten durfte. Mit rund 1250 Teilnehmern war es der bisher größte und am besten besuchte Österreichische Zahnärztekongress. Die Veranstaltung zeigte eindrucksvoll, wie wichtig den Kolleg(inn)en die unabhängige und von den Bedürfnissen unseres Berufsstandes getragene Fortbildung durch die ÖGZMK ist.
Die gute Zusammenarbeit zwischen Standespolitik und Fortbildung sowie die Kooperation zwischen den Länder- und Fachgesellschaften machen die Stärke der ÖGZMK aus. Eine hervorragende Basis für eine erfolgreiche Zukunft dieser traditionsreichen Einrichtung!

\section{Dr. Walter Keidel}

Präsident der ÖGZMK Salzburg

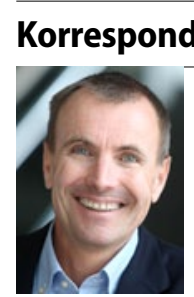

Dr. W. Keidel

Rochusgasse 4/LZÄK

5020 Salzburg

office@oegzmksalzburg.at 